\title{
Challenges and Future Consideration for Pharmacovigilance
}

\section{Vijay Kumar*}

GSK Consumer Healthcare Supply Chain, USA

\begin{abstract}
Pharmacovigilance is studying the safety and effectiveness of drugs and practice to minimize the possible risk associated with the use of drugs. It has developed significantly and will continue to develop in response to the new challenges it faces. With the rapid spread of information across the globe, increased communication across borders, easy access to variety of medicinal products and increasing public expectation of safety, there is a need for careful strategic planning adopting a holistic approach to meet the additional challenges. We need continuing and dynamic progress of all aspects of pharmacovigilance to improve public health and safety. There is a need for new global network to share information and intelligence about benefit and risk of medicinal products.
\end{abstract}

Keywords: Pharmacovigilance; Medicinal products; World Health Organization

\section{Introduction}

There is always a trade-off involved between the advantages and the possible risk with the use of pharmaceuticals. The risk is minimized when medicines of good quality, safety and efficacy are used rationally by an informed health professional and by patients. Pharmacovigilance helps in reducing the risk of harm by ensuring good quality medicines are used appropriately and expectations and concerns of patients are considered when therapeutic decisions are made. It ensures that health care providers and consumers have the information they need to make their decision about treatment. Pharmacovigilance has emerged as science to examine the safety and efficacy of drugs and other therapeutic products. The World Health Organization (WHO) defines pharmacovigilance as discipline to identify, review and understand drug adverse effects and taking required precautionary actions to curtail these drug linked problems [1]. Its importance has been critical to public health and has become an integral part of effective clinical practice. The first systemic international efforts to address drug safety were initiated in 1961, following the thalidomide tragedy [2,3]. When thalidomide was introduced, the pharmaceutical industry comprised of number of emerging competing companies and many of them were not dedicated to drug development. Also there were very few or no laws regulating drug development and marketing. Thalidomide use by pregnant mothers resulted in birth of thousands of congenitally deformed infants [2]. In response, efforts were initiated to address the drug safety issues by developing a system for detecting previously unknown or poorly understood adverse effects of medicines. Guidelines were developed to monitor drugs, foods and environmental contaminants for adverse reactions and toxicity. In the beginning these guidelines were to meet local and national needs. With globalization of world economy, free trade, increased communication across borders and increasing use of internet resulted in recognized need of a system, applicable internationally, to ensure safety of medicinal products. These efforts and recognition of system to monitor and promote the safety and effectiveness medicines lead to emergence of practice and science of pharmacovigilance.

Originally, pharmacovigilance purpose was to improve scientific understanding of safety profile of drugs and advise regulatory authorities. It has been limited largely to detection of unidentified adverse drug effects and support public health by providing reliable scientific information. However, there has been a growing awareness and realization that pharmacovigilance has the potential to progress beyond its confined limits and the scope of pharmacovigilance should be expanded to include new kind of safety concerns such as illegal sale and drug abuse over internet, increasing self-medication practices, widespread manufacture and sale of counterfeit medicines $[1,4,5]$. In recent times, the range of pharmacovigilance has been expanded to include, herbals medicines, blood products, biologics, medical devices and vaccines [5-7]. Additionally, there has been increasing use of traditional/herbal medicines outside the boundaries of the traditional culture and with other medicines that can cause the potential adverse interaction $[7,8]$. The pharmacovigilance faces new challenges by increasing demands and availability of range of new products. This article briefly describes some of the serious challenges pharmacovigilance faces and their potential implication.

\section{Drug Regulation}

There is a need for strong links within drug regulators to ensure that authorities are well briefed on everyday practice safety issue. There has been a substantial increase in number of clinical trials around the world. There is also an increasing partnership between academia and drug industries. This gives rise to widespread concern over ethical and scientific issues such as potential conflict of interest, unethical patient recruitment process, lack of competence for clinical trial monitoring, unethical clinical practice and poor reporting of adverse events [9]. Therefore drug regulators have increasing responsibility to ensure that right and health of patients and their commitments are protected. The growing intricacies of clinical trials also present further challenges to regulators [10]. Since clinical trials are usually carried out at multiple locations in different countries, local regulators are incognizant of investigator's proceedings at other international locations. Regulators often do not have capacity to efficiently ensure proper conduct of clinical trials. There is a need for standardized reporting systems for safety concerns arising during clinical trials and better coordination between ethics committees and investigators.

*Corresponding author: Vijay Kumar, GSK Consumer Healthcare Supply Chain 1500 Littleton Rd Parsippany, NJ 07054, USA, Tel: (973) 889-2351; E-mail: vijay.5.kumar@gsk.com

Received January 29, 2013; Accepted February 19, 2013; Published February 21,2013

Citation: Vijay Kumar (2013) Challenges and Future Consideration fo Pharmacovigilance. J Pharmacovigilance 1: 102. doi:10.4172/2329-6887.1000102

Copyright: @ 2013 Vijay Kumar. This is an open-access article distributed unde the terms of the Creative Commons Attribution License, which permits unrestricted use, distribution, and reproduction in any medium, provided the original author and source are credited. 
The process of new drug safety evaluation continues post marketing stage and regulators makes the final judgment regarding drug release considering detailed pharmacovigilance study. This is also important in introduction of generic medicines or safety profile of older medicines if there are new safety concerns. Some additional drug safety aspects such as excipients input to the safety profile, comparing safety profile of similar medicines and assessment of the adverse effects on human health of drug residues in animal needed to be included in long term effect study of medicines.

\section{Globalization}

The globalization of world economy has resulted in worldwide drug distribution and the increased exposure of massive populations to large volumes of medicines. The free trade and communication across borders and increasing use of internet have resulted in rapid spread of drug information and access to all medicinal products. These changes have resulted in new kind of safety concerns such as illegal sale of drug over internet, increases self-medication, public exposure to substandard products etc. The growing public awareness with global exposure has also heightened public expectation of safety and national pharmacovigilance centers are in further pressure to address all safety concerns [11]. There is a recognized need for competent and rapid communication between national drug regulatory centers as well as to other countries.

\section{Web-Based Sales and Promotional Activities}

Public health programs and media coverage aimed at increasing public awareness have made public in many countries to increasing influence health professional's prescribing and pattern of drug use. The Internet has encouraged the unrestrained sale of medicines across borders. Drug advertisement with unreliable claims is distributed internationally through the web. It ranges from prescription medicines, unregistered drugs and controlled substances to herbal medicines with suspicious quality and safety. Aggressive promotion by drug companies often results in unnecessary and irrational use of medicines by consumers [12]. Direct advertising to the consumer of prescription medicine has also become common in many countries. With this information patients feel competent to take greater responsibility for their own therapeutic decision without proper assistance from physician or pharmacist. It is welcome development in some situations; however available information is not always reliable and scientifically valid and produces serious health risks. This results in increasing self-medication, illicit availability of medicines over internet, and also physician overprescribing succumbing to patient demand [12]. These changes in drug use and practice have likely to have important consequences on public health and safety. Thus there is a need to determine how access to drug information can influence patient safety. Additionally, social and cultural characteristics of medicine use and patient's belief and concerns need to be further studied.

There is increasing public concern that close collaborations between academia and the pharmaceutical industries may unfavorably influence medical practice and clinical research. A disturbing development is "direct to consumer" advertising by drug makers, sellers and promoters with vested interests. The lack of reliability and accuracy of information provided to consumers may compromise patient care and safety. Furthermore, the internet provides a medium that makes communication possible across the borders even when direct advertising of prescription medicine to consumer is illegal. This makes national regulators irrelevant regarding advertising. These issues put forward the need for detailed monitoring of drug safety and scrutiny of advertising. It is necessary to ensure that promotional material provide truthful and unbiased information.

\section{Drug Utilization Pattern}

Drug consumption pattern are one of the major concern for drug safety. Route of administration is an important factor affecting drug safety. For examples, injectable medicines are more likely to be associated with a high risk of adverse effects. Irrational drug use, overdose, increasing self-medication practice and lack of regulatory control mechanism over drug sale increase the risk of adverse effects. Above all factors such as illiteracy, concomitant use of traditional medicine and increasing availability of substandard medicines in developing countries further worsen the risk. Thus pharmacovigilance programs should include public awareness and education of patients in rational use of medicine to ensure better health care in all countries. It is necessary that drug safety information should be easily available to public to ensure vital role of patient in rational safe use of medicine is understood.

\section{Cost}

While drugs are a prominent item in health budget, the cost of disease to society and money spent on detecting; preventing and managing drug's adverse effects are also included in cost consideration. Many studies have suggested the huge impact that poor product quality, adverse drug reactions and practice errors have on health care and patient health [13].

The number of national pharmacovigilance center has been increasing around the globe but situation in many developing countries is still different because of lacking budget support [14]. There are many countries where no formal system in place attributed to associated cost and limited resources. The debate over drug prices and intellectual properties rights are an important concern in developing countries. Additionally widespread use of potentially toxic medicines is an important issue in these countries with poor resources. For example, management of HIV in developing countries is illustrative of rising importance of pharmacovigilance in addressing health costs. The HIV control usually involves more than one potent drug, which are potentially toxic agents. In such situations, regular laboratory tests for liver function, hematology, viral load and viral resistance are essential to monitor safety and efficacy [15]. Moreover, the adverse effects such as hepatic, haematological, metabolic, and neurological disturbance are not uncommon and their treatment adds even further to the health budget $[15,16]$. Thus there is need to ensure that the existing centers are efficient and effective, their benefit on public health are evident.

\section{Biologics and Biosimilars}

Biologicals have specific characteristics, which complicate their safety assessment and require modified systems of safety monitoring. Biologicals are often prescribed for rare diseases, which make it difficult to include ample number of patients in the pre-approval clinical trials. Moreover, biologics are frequently used in multiple indications with different dosage regimens, which further complicate the exposure assessment of biological. They are often prescribed to patient with severe disease as second- or third-line therapy after the failure of standard treatment. Thus, pharmacovigilance for biologics involves additional challenges compared to small molecules.

Additional challenges are met for biosimilars, as they cannot be approved based on the same requirement that apply to generics. Biologics are made by a complicated production and purification 
process, which differs from one manufacturer to another [17]. Thus, it is very unlikely that manufacturer of the biosimilar will be able to precisely replicate the reference protein product as the original method is proprietary information of the owner of the reference product. It is recognized that small changes in the production and purification process of biologicals alter their safety profile as an adverse event can be related to a specific biological product $[18,19]$. Thus, regardless of the fact that the biosimilar and reference drug can show similar efficacy, the biosimilar can show different safety profile, which may influence the benefit-risk profile. It is essential to supervise the clinical safety and risk-benefit evaluation of biosimilars on a regular basis during the post-approval phase [20]. The safety profile of biosimilars should be established by identifying immunogenicity risk and appropriate post marketing supervision.

\section{Herbal and Traditional Medicines}

There is a common belief that "herbal" means "safe" and long-term use of traditional medicine assures its efficacy and safety. However, it is a misconception and irrational use of herbal and traditional medicines raises serious concerns about safety. Recently, traditional medicines have moved local boundaries and being manufactured for global use and there has been increasing number of cases of traditional and herbal medicines being adulterated or contaminated with other medicines [7]. Further, self-medication by consumers heightens the risk. The use of traditional and herbal medicine in combination with other medicine can cause very serious adverse drug interactions [21]. Therefore, these products should be included into regulatory framework like other pharmaceutical products. There should be standard of safety, quality and efficacy governing these products similar to other products intended for human use. But, lack of clear boundaries between foods and medicines get in the way in development of regulatory guidelines. The regulatory status of herbal products differs from country to country. These regulatory discrepancies between countries have serious implication for international availability of such products. If one county restricts the availability of an herbal product by prescription only, it may be obtained from health food shop or by mail order or internet from another country.

\section{Conclusion}

Pharmacovigilance has emerged over time as an essential part of public health program but encounters some crucial challenges. Its range is increasing with number and diversity of pharmaceutical products rising. The globalization of world economy and increased public exposure to large number of medicines, calls for a better coordination between drug regulators. There is a need for better integration of pharmacovigilance into clinical practice and public policy. A complicated and essential relationship exists between numbers of partners in drug safety monitoring. Sustained partnership and commitments are necessary between these partners to meet the future challenges in pharmacovigilance. The present pharmacovigilance systems need to be reviewed and evolved further to address future challenges.

\section{References}

1. The importance of Pharmacovigilance- Safety Monitoring of Medicinal Products (2002) WHO.
2. Kim JH, Scialli AR (2011) Thalidomide: the tragedy of birth defects and the effective treatment of disease. ToxicolSci 122: 1-6.

3. Collins TF (2006) History and evolution of reproductive and developmental toxicology guidelines. Curr Pharm Des 12: 1449-1465.

4. Edwards IR (2005) The WHO World Alliance for Patient Safety: a new challenge or an old one neglected? Drug Saf 28: 379-386.

5. Labadie J (2012) Forensic pharmacovigilance and substandard or counterfeit drugs. Int J Risk Saf Med 24: 37-39.

6. Graham JE, Borda-Rodriguez A, Huzair F, Zinck E (2012) Capacity for a global vaccine safety system: the perspective of national regulatory authorities. Vaccine 30: 4953-4959.

7. Shaw D, Graeme L, Pierre, Elizabeth D, Kelvin C (2012) Pharmacovigilance of herbal medicine. J Ethnopharmacol 140: 513-518.

8. Skalli S, Soulaymani Bencheikh R (2012) Safety monitoring of herb-drug interactions: a component of pharmacovigilance. Drug Saf 35: 785-791.

9. Härmark L, van Grootheest AC (2008) Pharmacovigilance: methods, recent developments and future perspectives. Eur J ClinPharmacol 64: 743-752.

10. Pal SN, Duncombe C, Falzon D (2013) Olsson S WHO Strategy for Collecting Safety Data in Public Health Programmes: Complementing Spontaneous Reporting Systems. Drug Saf 36: 75-81.

11. Almenoff JS (2007) Innovations for the future of pharmacovigilance. Drug Sa 30: 631-633.

12. Härmark L, van Grootheest K (2012) Web-based intensive monitoring: from passive to active drug surveillance. Expert Opin Drug Saf 11: 45-51.

13. Gautier S, Bachelet H, Bordet R, Caron J (2003) The cost of adverse drug reactions. Expert OpinPharmacother 4: 319-326.

14. Fernandopulle RB, Weerasuriya K (2003) What can consumer adverse drug reaction reporting add to existing health professional-based systems? Focus on the developing world. Drug Saf 26: 219-225.

15. Miller V, Nwokike J, Stergachis A (2012) Pharmacovigilance and global HIV AIDS. CurrOpin HIV AIDS 7: 299-304.

16. Licata A, Macaluso FS, Craxì A (2013) Herbal hepatotoxicity: a hidden epidemic. Intern Emerg Med 8: 13-22.

17. Joshi SR (2011) Biosimilar peptides: need for pharmacovigilance. J Assoc Physicians India 59: 44-47.

18. Abraham I, MacDonald K (2012) Clinical safety of biosimilar recombinant human erythropoietins. Expert Opin Drug Saf 11: 819-840.

19. Lee JF, Litten JB, Grampp G (2012) Comparability and biosimilarity: considerations for the healthcare provider. Curr Med Res Opin 28: 1053-1058.

20. Gascon P (2012) Presently available biosimilars in hematology-oncology: G-CSF. Target Oncol 7: S29-34.

21. Zhang L, Yan J, Liu X, Ye Z, Yang X, et al. (2012) Pharmacovigilance practice and risk control of Traditional Chinese Medicine drugs in China: current status and future perspective. J Ethnopharmacol 140: 519-525. 